\title{
Intravenous Tissue Plasminogen Activator Improves the Outcome in Very Elderly Korean Patients with Acute Ischemic Stroke
}

Jay Chol Choi, ${ }^{\mathrm{a}, *}$ Ji Sung Lee, ${ }^{\mathrm{b}, *}$ Tai Hwan Park, ${ }^{\mathrm{c}}$ Sang-Soon Park, ${ }^{\mathrm{c}}$ Yong-Jin Cho, ${ }^{\mathrm{d}}$ Jong-Moo Park, ${ }^{\mathrm{e}}$

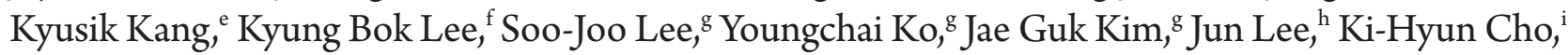
Joon-Tae Kim, ${ }^{i}$ Kyung-Ho Yu, ${ }^{j}$ Byung-Chul Lee, ${ }^{j}$ Mi-Sun Oh, Jae-Kwan Cha, ${ }^{k}$ Dae-Hyun Kim, ${ }^{k}$ Hyun-Wook Nah, ${ }^{\mathrm{k}}$ Dong-Eog Kim, ${ }^{1}$ Wi-Sun Ryu, ${ }^{1}$ Beom Joon Kim, ${ }^{\mathrm{m}}$ Hee-Joon Bae, ${ }^{\mathrm{m}}$ Wook-Joo Kim, ${ }^{\mathrm{n}}$ Dong-Ick Shin, ${ }^{o}$ Min-Ju Yeo, ${ }^{o}$ Sung Il Sohn, ${ }^{\mathrm{p}}$ Jeong-Ho Hong, ${ }^{\mathrm{p}}$ Juneyoung Lee, ${ }^{\mathrm{q}}$ Keun-Sik Hong ${ }^{\mathrm{d}}$

${ }^{a}$ Department of Neurology, Jeju National University, Jeju, Korea

${ }^{\mathrm{b} C l i n i c a l}$ Research Center, Asan Medical Center, Seoul, Korea

'Department of Neurology, Seoul Medical Center, Seoul, Korea

${ }^{\mathrm{d}}$ Department of Neurology, Ilsan Paik Hospital, Inje University, Goyang, Korea

'Department of Neurology, Eulji General Hospital, Eulji University, Seoul, Korea

${ }^{\mathrm{f}}$ Department of Neurology, Soonchunhyang University College of Medicine, Seoul, Korea

'Department of Neurology, Eulji University Hospital, Daejeon, Korea

h Department of Neurology, Yeungnam University Hospital, Daegu, Korea

iDepartment of Neurology, Chonnam National University Hospital, Gwangju, Korea

'Department of Neurology, Hallym University Sacred Heart Hospital, Anyang, Korea

${ }^{k}$ Department of Neurology, Dong-A University College of Medicine, Busan, Korea

${ }^{1}$ Department of Neurology, Dongguk University Ilsan Hospital, Goyang, Korea

${ }^{m}$ Department of Neurology, Seoul National University Bundang Hospital, Seoul National University College of Medicine, Seongnam, Korea

"Department of Neurology, Ulsan University Hospital, Ulsan, Korea

${ }^{\circ}$ Department of Neurology, Chungbuk National University Hospital, Cheongju, Korea

PDepartment of Neurology, Keimyung University Dongsan Medical Center, Daegu, Korea

${ }^{q}$ Department of Biostatistics, Korea University College of Medicine, Seoul, Korea

Background and Purpose In a recent pooled analysis of randomized clinical trials (RCTs), intravenous tissue plasminogen activator (TPA) improves the outcome in patients aged $\geq 80$ years. However, it is uncertain whether the findings are applicable to clinical practice in Asian populations. Methods From a multicenter stroke registry database of Korea, we identified patients with acute ischemic stroke who were aged $\geq 80$ years. Using multivariable analysis and propensity score (PS)-matched analyses, we assessed the effectiveness and safety of intravenous TPA within 4.5 hours.

Results Among 2,334 patients who met the eligible criteria, 236 were treated with intravenous TPA (mean age, 83 \pm 5 ; median NIHSS, 13 [IOR, 8-17]). At discharge, the TPA group compared to the no-TPA group had a favorable shift on the modified Rankin Scale (mRS) score (multivariable analysis, OR [95\% Cl], 1.51 [1.17-1.96], $P=0.002$; PS-matched analysis, 1.54 [1.17-2.04], $P=0.002$ ) and was more likely to achieve mRS 0-1 outcome (multivariable analysis, 2.00 [1.323.03], $P=0.001$; PS-matched analysis, 1.59 [1.04-2.42], $P=0.032$ ). TPA treatment was associated with an increased risk of symptomatic intracranial hemorrhage (multivariable analysis, 5.45 [2.80-10.59], $P<0.001$; PS-matched analysis, 4.52 [2.24-9.13], $P<0.001)$, but did not increase the
Correspondence: Keun-Sik Hong Department of Neurology, llsan Paik Hospital, Inje University, 170 Juhwa-ro, Ilsanseo-gu, Goyang 10380, Korea Tel: +82-31-910-7680 Fax: +82-31-910-7368

E-mail: nrhks@paik.ac.kr

Received: February 17, 2015

Revised: April 20, 2015

Accepted: April 29, 2015

*Jay Chol Choi and Ji Sung Lee contributed equally to the manuscript as the first author.

This study was supported from the Korea Healthcare Technology REtD Project. Ministry of Health, Republic of Korea (HI10C2020).

The authors have no financial conflicts of interest. 
in-hospital mortality (multivariable analysis, 0.86 [0.50-1.48], $P=0.58$; PS-matched analysis, 0.88 [0.52-1.47], $P=0.61)$.

Conclusions In the setting of clinical practice, intravenous TPA within 4.5 hours improved the functional outcome despite an increased risk of symptomatic intracranial hemorrhage in very elderly Korean patients. The findings, consistent with those from pooled analysis of RCTs, strongly support the use of TPA for this population.

Keywords Elderly; Ischemic stroke; Thrombolytic therapy; Outcome assessment

\section{Introduction}

Stroke population as well as global population is aging. In developed countries, the proportion of elderly patients among stroke population increased, ${ }^{2}$ and more than $30 \%$ of stroke patients were aged $\geq 80$ years and $7.2 \%$ to $14.2 \%$ were aged $\geq 85$ years. ${ }^{3-6}$ However, very elderly patients were excluded from or substantially under-represented in earlier intravenous tissue-plasminogen activator (TPA) trials. ${ }^{7-11}$ In the third International Stroke Trial (IST3) where $53 \%$ of 3,035 patients enrolled were aged $>80$ years, the benefit of TPA was greater in patients aged $>80$ years than in those $\leq 80$ years, but the result did not reach statistical significance. ${ }^{12}$ In an updated pooled analysis of randomized clinical trials (RCTs), ${ }^{13}$ intravenous TPA significantly increased the excellent outcome defined by modified Rankin Scale (mRS) score 0-1 in patients aged $>80$ years as well as those $\leq 80$ years.

Although many countries have not formally approved the use of TPA for patients aged over 80 years, experienced centers have treated very elderly patients with intravenous TPA if eligible. In accordance with clinical trial results, data from real world practice also showed that the TPA treatment improved outcome in patients aged $>80$ years although their outcomes were worse than observed in those aged $\leq 80$ years. However, the data were generally driven by white populations. ${ }^{14,15}$ Compared to white populations, Asian populations are at higher risk of hemorrhagic complications after TPA treatment in acute ischemic stroke. ${ }^{16}$ For very elderly Asian patients, data on the effectiveness and safety of TPA treatment in the setting of clinical practice are limited. In Japanese and Taiwanese studies, symptomatic intracranial hemorrhage (SICH) was not substantially and significantly increased with TPA, but most patients were treated with a lower dose. ${ }^{17,18}$ In addition, the benefit was not consistent; TPA treatment improved the outcome in the Japanese study, ${ }^{17}$ but not in the Taiwanese study. ${ }^{18}$

In Korea, about $8 \%$ of patients with acute ischemic stroke are being treated with TPA, ${ }^{19}$ and the rate of thrombolytic therapy is increasing in stroke centers. ${ }^{20}$ Despite the lack of marketing authorization for patients aged $>80$ years in Korea, most cen- ters provide TPA therapy to eligible patients with the standard dose of $0.9 \mathrm{mg} / \mathrm{kg}$. The current study aims to assess the effectiveness and safety of TPA in very elderly Korean patients using data from a prospective stroke registry.

\section{Methods}

\section{Database and subjects}

The Clinical Research Center for Stroke-5 (CRCS-5) registry is a prospective registry of patients with acute ischemic stroke admitted to 15 academic centers in Korea (http://www.stroke-crc. or.kr). The detailed information on the registry including the design, fields, and characteristics of data have been published previously. ${ }^{20,21}$ The CRCS-5 registry was approved by the Institutional Review Boards of all participating centers. The informed consent from individual patients or their legally authorized representatives was waived by the relevant institutional review boards because the registry aimed to monitor and improve the quality of stroke care and a computer-assisted de-identification system ensured the anonymity of individual patients during data collection. This study was additionally approved by the institutional review board of Ilsan Paik Hospital.

To the CRCS-5 dataset between April 2008 and November 2013, we applied following inclusion criteria; (1) age $\geq 80$ years, (2) evidence of acute ischemic stroke on neuroimaging, and (3) admitted to the hospitals within 7 days from symptom onset. The exclusion criteria were (1) patients with pre-stroke mRS score of $>1,(2)$ patients who were treated with intraarterial or combined intravenous and intraarterial thrombolytic therapy, (3) patients who were treated with intravenous TPA at outside hospitals, and (4) unavailability of discharge mRS.

\section{Data collection}

For this study, we extracted the following data from the main registry: (1) age, sex, pre-stroke functional disability measured by $\mathrm{mRS}$ score, systolic and diastolic blood pressure at admission, and onset to arrival time; (2) laboratory findings (fasting blood glucose, fasting total cholesterol, fasting LDL cholesterol, creati- 
nine, and hemoglobin); (3) vascular risk factors (hypertension, diabetes mellitus, hyperlipidemia, smoking, atrial fibrillation, and prior history of stroke and coronary artery disease); (4) medications prior to the index stroke (antiplatelet agents, statins and oral anticoagulants); (5) characteristics of the index stroke including initial stroke severity measured by the National Institutes of Health Stroke Scale (NIHSS) score, symptomatic stenosis or occlusion of intracranial or extracranial artery (SYSO); (6) thrombolytic treatment (use of TPA, dosage of TPA, and onset to treatment time); (7) outcomes after the index stroke (mRS scores at discharge and at 3 months after the stroke and SICH).

Ischemic stroke was classified as large-artery atherosclerosis (LAA), small-vessel occlusion (SVO), cardioembolism (CE), stroke of undetermined etiology (UDE) or stroke of other determined etiology (ODE) according to the TOAST criteria. ${ }^{22} \mathrm{SICH}$ was defined by the European Cooperative Acute Stroke Study (ECASS) III criteria of any apparently extravascular blood in the brain or within the cranium associated with a worsening of NIHSS score $\geq 4$ or leading to death. ${ }^{9}$ SYSO was assessed using computed tomography angiography, magnetic resonance angiography, or conventional catheter angiography. For intracranial arteries, significant stenosis was defined as more than $50 \%$ stenosis of the artery compared with proximal or distal normal segment. ${ }^{23}$

\section{Outcome measurement}

The primary outcome was the distribution of $\mathrm{mRS}$ scores at discharge and the secondary outcomes were the proportions of mRS 0-1 and mRS 0-2 at discharge, SICH, and in-hospital mortality. For sensitivity analysis, we assessed the distribution of $\mathrm{mRS}$ scores at 3 months after the index stroke.

\section{Statistical analysis}

Values are presented as mean \pm standard deviation (SD), median (interquartile range $[\mathrm{IQR}]$ ) for continuous variables, or as the number (\%) of subjects for categorical variables. Comparisons of baseline characteristics between the TPA patients and untreated patients were made using the Pearson chi-square test, Student $t$ test or Wilcoxon rank sum test according to the type of variable.

For the primary outcome, we compared the overall distribution of discharge $\mathrm{mRS}$ scores between the two groups. The odds ratio (OR) and $95 \%$ confidence interval $(\mathrm{CI})$ were calculated to estimate the probabilities of favorable one level shift on the mRS score with TPA treatment. The Cochran-Mantel-Haenszel test was used for unadjusted shift analysis, and the ordinal logistic regression analysis was used to adjust covariates. For the secondary outcomes, we used Chi-square test or Fisher's exact test for unadjusted analysis and binary logistic regression analysis for covariates adjustment.

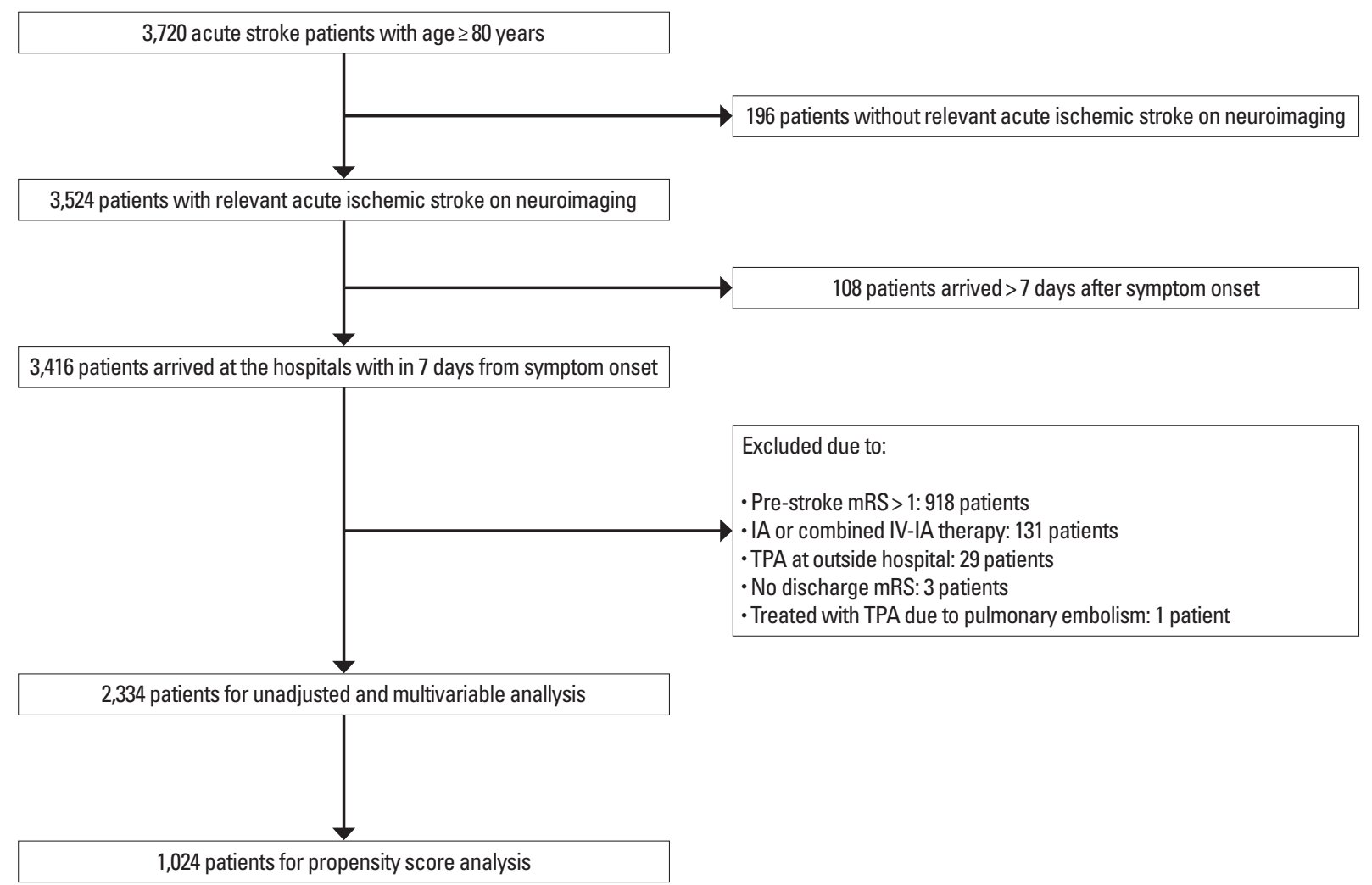

Figure 1. Study flow diagram. mRS, modified Rankin Scale; TPA, tissue plasminogen activator; IA, intraarterial; IV-IA, intravenous-intraarterial. 
Because the baseline characteristics of the two groups were expected to differ significantly, particularly for stroke severity, we conducted propensity score (PS)-matched analysis in addition to multivariable analysis. To estimate PS, the binary logistic regression with the TPA treatment as a dependent variable was used, and the model included all relevant pretreatment characteristics related to the TPA treatment. After obtaining the PS, we used a greedy-matching algorithm with maximal width of calipers being 0.2 standard deviations of the logit of the estimated PS. ${ }^{24}$ To check the baseline imbalances between the two groups after the PS matching, absolute standardized differences (ASDs) was computed for each covariate.

After the PS matching, we performed outcome analysis using ordinal or logistic regression analysis based on the generalized estimation equation method. Clinically important covariates such as age, sex, pre-stroke mRS, initial NIHSS score, and fasting blood glucose level were entered into the final model regardless of their ASD values. Covariates with an ASD $>0.1$ were further adjusted in PS-matched analysis. In all analyses, a $P$ value $<0.05$ was considered statistically significant. SAS computer software (Version 9.3, SAS Institute, Cary, NC, USA) was used for the statistical analyses.

Table 1. Baseline characteristics of TPA patients and untreated patients before and after PS matching

\begin{tabular}{|c|c|c|c|c|c|c|}
\hline & \multicolumn{3}{|c|}{ Unmatched cohort } & \multicolumn{3}{|c|}{ PS-matched cohort } \\
\hline & No TPA $(n=2,098)$ & TPA $(n=236)$ & $P$ value ${ }^{*}$ & № TPA (n=788) & TPA $(n=236)$ & $P$ value ${ }^{\dagger}$ \\
\hline \multicolumn{7}{|l|}{ Demographics } \\
\hline Age & $84.0 \pm 3.6$ & $83.5 \pm 3.2$ & 0.031 & $83.5 \pm 3.3$ & $83.5 \pm 3.2$ & 0.94 \\
\hline Male & $842(40.1)$ & $93(39.4)$ & 0.83 & $310(39.3)$ & $93(39.4)$ & 0.81 \\
\hline Pre-stroke mRS & & & 0.63 & & & 0.98 \\
\hline 0 & $1,835(87.5)$ & $209(88.6)$ & & 700 (88.8) & 209 (88.6) & \\
\hline 1 & $263(12.5)$ & $27(11.4)$ & & $88(11.2)$ & $27(11.4)$ & \\
\hline \multicolumn{7}{|l|}{ Admission characteristics } \\
\hline Onset to arrival time (hour) & $15.6(5.5-40.5)$ & $1.5(0.8-2.5)$ & $<0.001$ & $12.4(5.0-29.1)$ & $1.5(0.8-2.5)$ & $<0.001$ \\
\hline Clear onset & $1,357(64.7)$ & $195(82.66)$ & $<0.001$ & $428(54.3)$ & $195(82.66)$ & $<0.001$ \\
\hline \multicolumn{7}{|l|}{ Risk factors } \\
\hline Hypertension & $1,567(74.7)$ & $173(73.3)$ & 0.64 & $582(73.9)$ & $173(73.3)$ & 0.95 \\
\hline $\mathrm{DM}$ & $539(25.7)$ & 54 (22.9) & 0.35 & $204(25.9)$ & 54 (22.9) & 0.58 \\
\hline Hyperlipidemia & $584(27.8)$ & $57(24.2)$ & 0.23 & $189(24.0)$ & $57(24.2)$ & 0.87 \\
\hline Smoking & $494(23.5)$ & $54(22.9)$ & 0.82 & $188(23.9)$ & $54(22.9)$ & 0.81 \\
\hline Atrial fibrillation & $597(28.5)$ & $117(49.6)$ & $<0.001$ & $380(48.2)$ & $117(49.6)$ & 0.53 \\
\hline History of stroke & $404(19.3)$ & $40(16.9)$ & 0.39 & $128(16.2)$ & $40(16.9)$ & 0.91 \\
\hline History of CAD & $216(10.3)$ & $26(11.0)$ & 0.73 & $89(9.9)$ & $26(11.0)$ & 0.84 \\
\hline \multicolumn{7}{|l|}{ Laboratory findings } \\
\hline $\mathrm{SBP}(\mathrm{mmHg})$ & $146.9 \pm 27.1$ & $151.7 \pm 29.0$ & 0.012 & $150.6 \pm 29.5$ & $151.7 \pm 29.0$ & 0.81 \\
\hline Hemoglobin (g/dL) & $12.9 \pm 1.9$ & $12.8 \pm 1.5$ & 0.52 & $12.8 \pm 2.0$ & $12.8 \pm 1.5$ & 0.84 \\
\hline Fasting glucose (mg/dL) & $114.0 \pm 40.2$ & $121.5 \pm 40.7$ & 0.006 & $120.0 \pm 43.5$ & $121.5 \pm 40.7$ & 0.98 \\
\hline LDL cholesterol (mg/dL) & $107.6 \pm 34.9$ & $107.5 \pm 33.2$ & 0.96 & $106.9 \pm 35.0$ & $107.5 \pm 33.2$ & 0.88 \\
\hline Creatinine (mg/dL) & $1.1 \pm 0.9$ & $1.0 \pm 0.6$ & 0.76 & $1.0 \pm 0.7$ & $1.0 \pm 0.6$ & 0.94 \\
\hline \multicolumn{7}{|l|}{ Stroke characteristics } \\
\hline Baseline NIHSS score, median (IOR) & $4(2-9)$ & $13(8-17)$ & $<0.001$ & $10(4-17)$ & $13(8-17)$ & 0.77 \\
\hline Stroke subtype & & & $<0.001$ & & & 0.60 \\
\hline LAA & $762(36.3)$ & $57(24.2)$ & & $204(25.9)$ & $57(24.2)$ & \\
\hline SVO & $281(13.4)$ & $8(3.4)$ & & $20(2.5)$ & $8(3.4)$ & \\
\hline CE & $568(27.1)$ & $118(50.0)$ & & $372(47.2)$ & $118(50.0)$ & \\
\hline UDE or ODE & $487(23.2)$ & $53(22.5)$ & & $192(24.4)$ & $53(22.5)$ & \\
\hline SYSO & $979(46.7)$ & $155(65.7)$ & $<0.001$ & $496(62.9)$ & $155(65.7)$ & 0.98 \\
\hline \multicolumn{7}{|l|}{ Pre-stroke medication } \\
\hline Antiplatelet & $639(30.5)$ & $79(33.5)$ & 0.34 & $253(32.1)$ & $79(33.5)$ & 0.86 \\
\hline Anticoagulant & $85(4.1)$ & $6(2.5)$ & 0.26 & $28(3.6)$ & $6(2.5)$ & 0.58 \\
\hline Statin & $290(13.8)$ & $27(11.4)$ & 0.31 & $92(11.7)$ & $27(11.4)$ & 0.99 \\
\hline
\end{tabular}

Values are number of patients (\%), mean $\pm S D$ or median (IQR) unless otherwise indicated.

${ }^{*} P$ values are calculated by Pearson chi-square test, Fisher's exact test, Student's $t$ test or Wilcoxon rank sum test as appropriate; ${ }^{\dagger} P$ values are calculated by conditional logistic regression.

PS, propensity score; TPA, tissue plasminogen activator; mRS, modified Rankin Scale; DM, diabetes mellitus; CAD, coronary artery disease; SBP, systolic blood pressure; NIHSS, National Institutes of Health Stroke Scale; IQR, interquartile range; LAA, large-artery atherosclerosis; SVO, small-vessel occlusion; CE, cardioembolism; UDE, stroke of undetermined etiology; ODE, stroke of other determined etiologies; and SYSO, symptomatic stenosis or occlusion of intracranial or extracranial artery. 


\section{Results}

Of 3,720 patients with acute ischemic stroke $\geq 80$ years old enrolled in the CRCS-5 registry between April 2008 and Nov 2013, we excluded 1,386 patients in the following order: (1) 196 patients without relevant acute ischemic stroke on neuroimaging; (2) 108 patients who admitted to the participating hospitals beyond 7 days from symptom onset; (3) 918 patients with a pre-stroke mRS score $>1$; (4) 131 patients treated with intraarterial or combined intravenous and intraarterial thrombolysis; (5) 29 patients who were treated with TPA at outside hospitals; (6) 3 patients in whom discharge $\mathrm{mRS}$ scores were unavailable; and (7) 1 patient who were treated with intravenous TPA due to pulmonary embolism (Figure 1).

The final study cohort for the current analysis included 2,334 patients and the mean age of the patients was 83.9 years $(S D, 3.5)$ with $40.1 \%$ being men. The median delay from onset-to-door was 12.7 hours (IQR, 3.7-34.1) and the median NIHSS score at presentation was 5 (IQR, 2-11). LAA (35.1\%) was the most

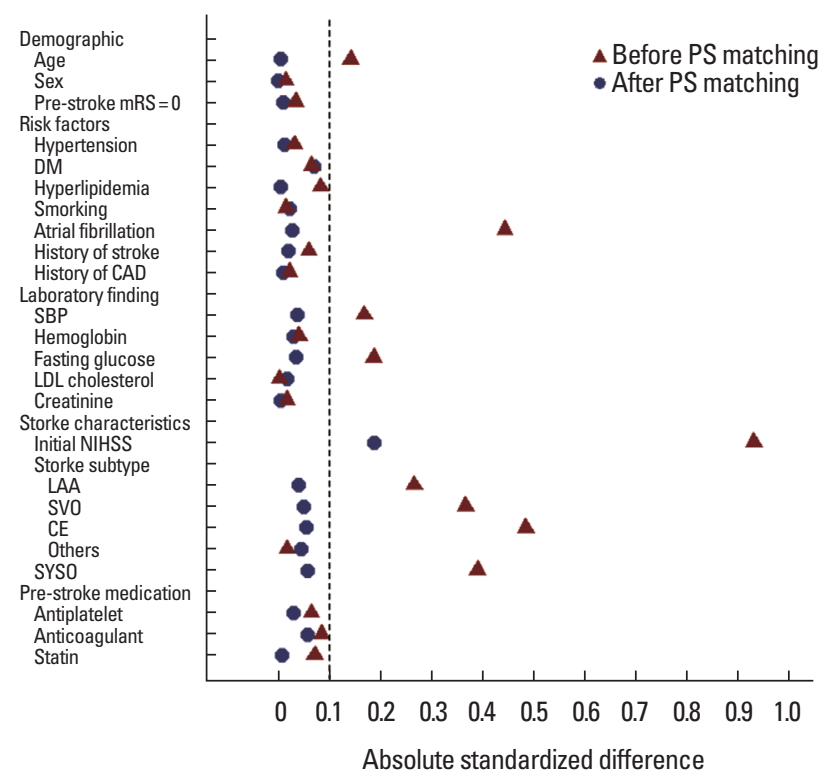

Figure 2. Absolute standardized differences of covariates before and after propensity score matching common ischemic stroke subtype, followed by CE (29.4\%), UDE or ODE (23.1\%) and SVO (12.4\%). Other baseline characteristics are presented in Table 1.

Of the 2,334 patients, 236 (10.1\%) were treated with TPA. The median onset-to-treatment and door-to-treatment time were 131 minutes (IQR, 94-180) and 40 minutes (IQR, 30-52) respectively: 76\% (179 patients) were treated within 3 hours, 19\% (46 patients) between the 3-4.5 hours, and 5\% (11 patients) beyond the 4.5 hours window. Of the 236 patients, 187 patients $(79.2 \%)$ received $0.9 \mathrm{mg} / \mathrm{kg}$ of TPA. The TPA group compared with the no-TPA group had a higher NIHSS score at presentation (median NIHSS [IQR], 13 [8-17] versus 4 [2-9]; $P<0.001)$. In addition, the TPA group versus the no-TPA group was more likely to be younger and to have atrial fibrillation, SYSO, higher fasting blood glucose, and higher initial systolic blood pressure. The distribution of ischemic stroke subtypes differed between the two groups with $\mathrm{CE}$ being the most common in the TPA group whereas LAA being the most frequent subtype in the no-TPA group. At discharge, 44 (18.6\%) among the 236 TPA-treated patients and 603 (28.7\%) among the 2,098 untreated patients achieved an excellent functional outcome ( $\mathrm{mRS}$ $0-1)$. Using the PS, 236 patients treated with TPA were matched to 788 untreated patients (1:2 in 53 pairs, $1: 3$ in 50 pairs, and 1:4 in 133 pairs). After the PS matching, most baseline characteristics were well balanced between the two arms, but the ASD value for the initial NIHSS score remained $>0.1$, indicating a residual imbalance even after the PS matching (Figure 2).

For the primary outcome, on unadjusted analysis, the TPA group had a worse discharge $\mathrm{mRS}$ score distribution compared with the no-TPA group (OR 0.48; 95\% CI, 0.38-0.61; $P<0.001$ ). However, on multivariable logistic regression analysis, the TPA group compared to the no-TPA group had a statistically significant favorable shift on the discharge $\mathrm{mRS}$ score distribution (OR 1.51 ; 95\% CI, 1.17-1.96; $P=0.002$ ). After the PS matching, the TPA effect of shifting the functional outcome towards better $\mathrm{mRS}$ outcome categories remained significant and was similar to the result of multivariable analysis (OR 1.54; 95\% CI, 1.17-2.04; $P=0.002$ ) (Table 2 and Figure 3).

For secondary outcomes, before covariates adjustment and PS

Table 2. Effectiveness of intravenous TPA on discharge mRS outcome

\begin{tabular}{|c|c|c|c|c|c|c|c|c|c|}
\hline & \multicolumn{3}{|c|}{ Shift analysis } & \multicolumn{3}{|c|}{ mRS 0-1 proportion } & \multicolumn{3}{|c|}{ mRS 0-2 proportion } \\
\hline & $O \mathrm{R}^{*}$ & $95 \% \mathrm{Cl}$ & $P$ value & $\mathrm{OR}$ & $95 \% \mathrm{Cl}$ & $P$ value & $\mathrm{OR}$ & $95 \% \mathrm{Cl}$ & $P$ value \\
\hline Unadjusted analysis & 0.48 & $0.38-0.61$ & $<0.001$ & 0.57 & $0.40-0.80$ & 0.001 & 0.49 & $0.36-0.66$ & $<0.001$ \\
\hline Multivariable analysis $^{\dagger}$ & 1.51 & $1.17-1.96$ & 0.002 & 2.00 & $1.32-3.03$ & 0.001 & 2.01 & $1.36-2.95$ & $<0.001$ \\
\hline PS-matched analysis ${ }^{\ddagger}$ & 1.54 & $1.17-2.04$ & 0.002 & 1.59 & $1.04-2.42$ & 0.032 & 1.97 & $1.31-2.99$ & 0.001 \\
\hline
\end{tabular}

*Odd ratios for achieving a favorable shifting of one level on the mRS score; ${ }^{\dagger}$ Adjusted for age, sex, previous mRS, initial NIHSS, fasting glucose, atrial fibrillation, SBP, TOAST, and SYSO; 'PS matching samples included 236 pairs (1:2, 53 pairs; 1:3, 50 pairs; 1:4, 133 pairs) and were adjusted for age, sex, previous mRS, initial NIHSS, and fasting glucose. TPA, tissue plasminogen activator; mRS, modified Rankin Scale; OR, odds ratio; Cl, confidence interval. 


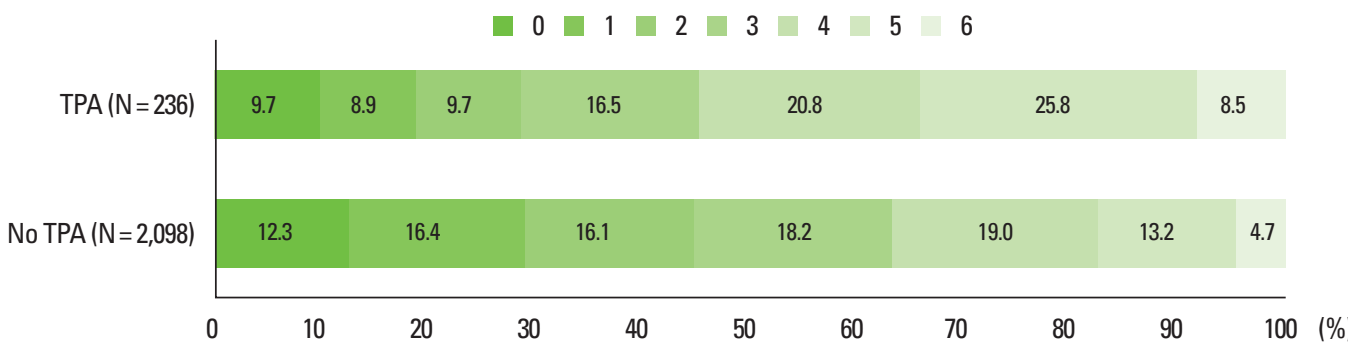

A

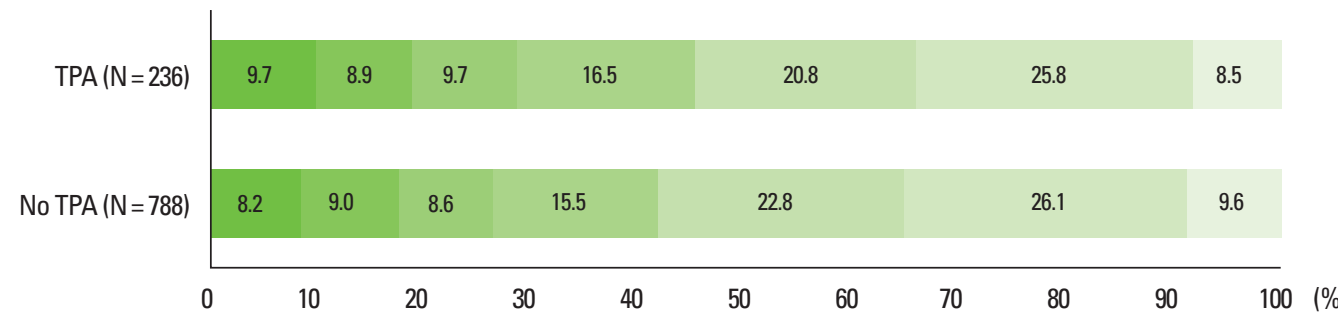

Figure 3. Distribution of discharge modified Rankin Scale score before (A) and after (B) propensity score matching.

Table 3. Risks of SICH and in-hospital mortality with TPA

\begin{tabular}{|c|c|c|c|c|c|c|}
\hline & & $\mathrm{SICH}$ & & & ospital mort & \\
\hline & $\mathrm{OR}$ & $95 \% \mathrm{Cl}$ & $P$ value & $\mathrm{OR}$ & $95 \% \mathrm{Cl}$ & $P$ value \\
\hline Unadjusted analysis & 9.54 & 4.89-18.61 & $<0.001$ & 1.87 & $1.13-3.08$ & 0.014 \\
\hline Multivariable analysis* & 5.45 & $2.80-10.59$ & $<0.001$ & 0.85 & $0.50-1.48$ & 0.58 \\
\hline PS matching $^{\dagger}$ & 4.52 & $2.24-9.13$ & $<0.001$ & 0.77 & $0.52-1.47$ & 0.61 \\
\hline
\end{tabular}

*Adjusted for age, sex, previous mRS, initial NIHSS, fasting glucose, atrial fibrillation, SBP, TOAST, and SYSO; 'PS matching samples included 236 pairs (1:2, 53 pairs; 1:3, 50 pairs; 1:4, 133 pairs) and were adjusted for age, sex, previous mRS, initial NIHSS, and fasting glucose.

SICH, symptomatic intracranial hemorrhage; TPA, tissue plasminogen activator; OR, odds ratio; Cl, confidence interval; PS, Propensity score.

Table 4. Effectiveness of TPA on 3-month mRS score distribution

\begin{tabular}{lccr} 
& \multicolumn{3}{c}{ Overall $\mathrm{mRS}$ at 3 months } \\
\cline { 2 - 4 } & OR & $95 \% \mathrm{Cl}$ & \multicolumn{1}{c}{ Pvalue } \\
\hline Unadjusted analysis $^{*}$ & 0.47 & $0.37-0.61$ & $<0.001$ \\
Multivariable analysis* $^{*}$ & 1.38 & $1.05-1.82$ & 0.021 \\
PS matching $^{\dagger}$ & 1.36 & $1.01-1.83$ & 0.044 \\
\hline
\end{tabular}

${ }^{*}$ Adjusted for age, sex, previous mRS, initial NIHSS, fasting glucose, atrial fibrillation, SBP, TOAST, and SYSO; 'PS matching samples included 236 pairs (1:2, 53 pairs; 1:3, 50 pairs; 1:4, 133 pairs) and were adjusted for age, sex, previous mRS, initial NIHSS, and fasting glucose.

TPA, tissue plasminogen activator; OR, odds ratio. Cl, confidence interval; PS, Propensity score.

matching, the proportions of discharge mRS 0-1 and mRS 0-2 outcomes were lower in the TPA group than in the no-TPA groups. However, on multivariable analysis, the TPA group was more likely to achieve mRS 0-1 (OR 2.00; 95\% CI, 1.32-3.03; $P=0.001$ ) and mRS 0-2 (OR 2.01; 95\% CI, 1.36-2.95; $P<$ 0.001 ) outcomes at discharge (Table 2). For the PS-matched cohort, the TPA group compared to the no-TPA group had significantly higher proportions of mRS $0-1$ ( $18.6 \%$ vs. $17.3 \%$; OR 1.59 ; $95 \% \mathrm{CI}, 1.04-2.42 ; P=0.032)$ and $\mathrm{mRS} 0-2(28.4 \%$ vs. $25.9 \%$; OR 1.97 ; $95 \% \mathrm{CI}, 1.31-2.99$; $P=0.001)$ outcomes at dis- charge (Table 2).

SICH was found in 18 (7.6\%; 95\% CI, 4.6\%-11.8\%) among the $236 \mathrm{TPA}$-treated patients and 18 patients $(0.9 \%$; $95 \% \mathrm{CI}$, $0.5 \%-1.4 \%$ ) among the 2,098 untreated patients (OR, 9.54; $95 \%$ CI, 4.89-18.61; $P<0.001)$. TPA treatment was associated with an increased risk of SICH on multivariable logistic regression analysis (OR, 5.45; 95\% CI, 2.80-10.59; $P<0.001)$ and PS-matched analysis (OR, 4.52; 95\% CI, 2.24-9.13; $P<0.001$ ) (Table 3). For mortality, unadjusted analysis showed that the in-hospital mortality was higher in the TPA group than in the no-TPA group (8.5\% vs. $4.7 \%$ ). However, after adjusting for covariates, it did not differ between the two groups (OR, 0.86 ; $95 \% \mathrm{CI}, 0.50-1.48 ; P=0.58$ ). For the PS-matched cohort, the in-hospital mortality rate was not different: $8.5 \%$ for the TPA group and $9.6 \%$ for the no-TPA group (OR, 0.88; 95\% CI, 0.52-1.47; $P=0.62$ ) (Table 3).

The $\mathrm{mRS}$ scores at 3 months were available in 1,909 patients (81.8\%) and were analyzed as a sensitivity analysis. At 3 months, TPA favorably and significantly shifted the mRS score distribution in the multivariable and PS-matched analyses, and the findings were generally consistent with those observed from the discharge mRS outcome (Table 4 and Figure 4 ). 


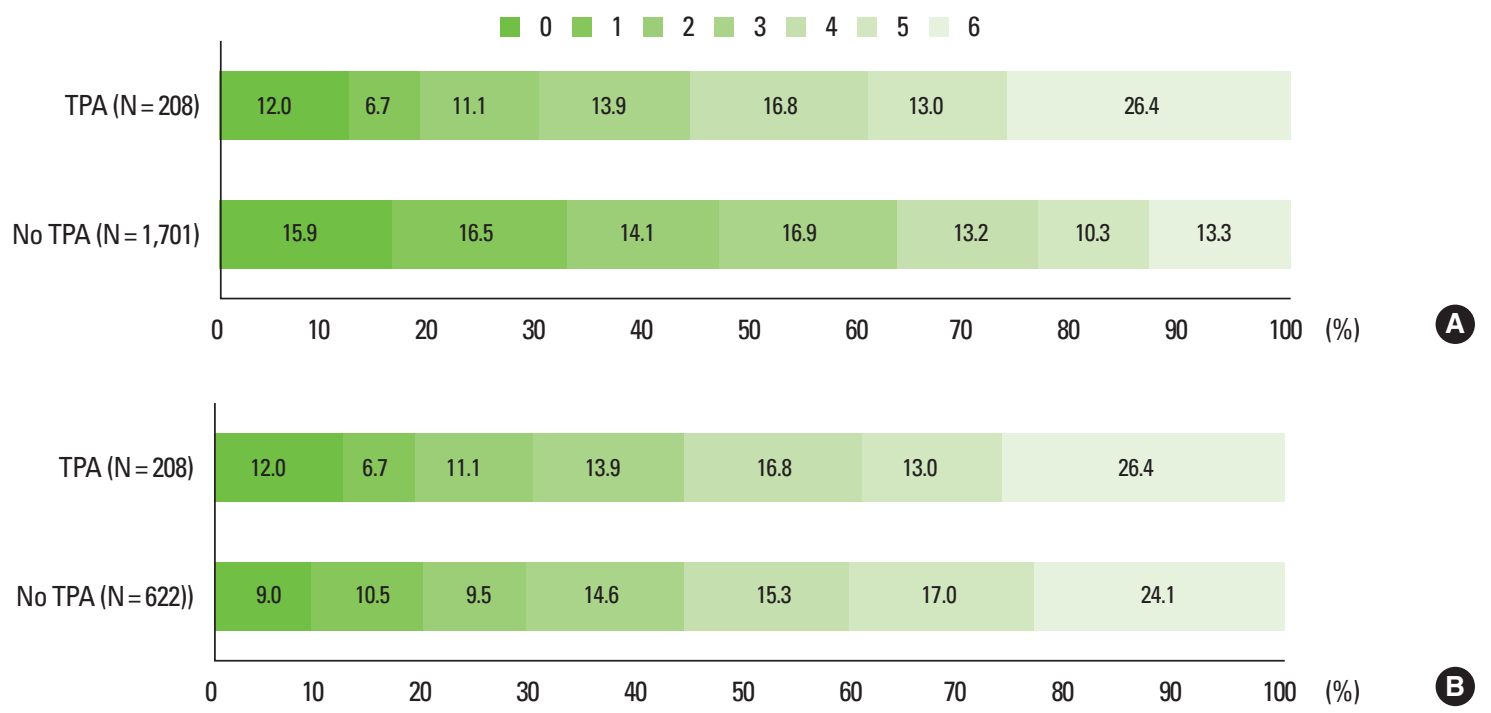

Figure 4. Distribution of 3-month modified Rankin Scale score before (A) and after (B) propensity score matching.

\section{Discussion}

In this study of very elderly Korean patients with acute ischemic stroke, there was a significant outcome improvement with intravenous TPA treatment in routine clinical practice. The TPA treatment within 4.5 hours resulted in about 1.5 -fold increased odds of one level shift towards better mRS outcome categories, 1.6- to 2.0-fold increased odds of an excellent outcome of $\mathrm{mRS}$ $0-1$, and almost 2.0-fold increased odds of a good outcome of mRS 0-2. Although the risk of SICH increased with TPA by approximately 4.5 - to 5.5 -fold, it did not translate into an increase in in-hospital mortality.

Our findings are in accordance to and extend data regarding the TPA benefit from earlier pooled analysis of RCTs and observational studies. In our PS-matched analysis, the increased odds (95\% CI) of mRS 0-1 outcome with TPA was 1.59 (1.04-2.42), whereas it was $1.56(1.17-2.08)$ in patients aged $>80$ years in the pooled-analysis of RCTs. ${ }^{13}$ In addition, the TPA effect on shift analysis was similar between our results (1.51 [1.17-1.96] on multivariable analysis; 1.54 [1.17-2.04] on PS matching analysis) and that (1.4 [1.3-1.6]) found in a case-control study of the International Stroke Thrombolysis Registry (SITS-ISTR) and Virtual International Stroke Trials Archive (VISTA) datasets. ${ }^{15}$ Therefore, the TPA benefit observed in clinical practice of very elderly Korean patients was quite comparable to those obtained in the clinical trials and an observational study largely driven from white populations. Among Asian studies, a Japanese registry study showed that the odds (95\% CI) of achieving mRS 0-2 outcome at discharge with TPA treatment was 2.23 (1.16-4.29) on PS-matched analysis, ${ }^{17}$ which was comparable to our odds of 1.97 (1.31-2.99) seen on the similar analysis. The findings from the two Japanese and Korean studies with different baseline characteristics and TPA dose $(0.6 \mathrm{mg} / \mathrm{kg}$ in the Japanese study versus $0.9 \mathrm{mg} / \mathrm{kg}$ in $79.2 \%$ of our patients) strongly suggest the TPA benefit in very elderly Asian patients.

Patients with advanced age are at high risk of $\mathrm{SICH}$, which potentially mitigates the TPA benefit in very elderly patients. In the current study, the TPA treatment was associated with an increased risk of SICH as expected. The rate of SICH per the ECASS III definition was $7.6 \%$, which appears higher than observed in the comparable population in the SITS-ISTR registry (2.5\% per the SITS-MOST definition) ${ }^{15}$ the Japanese study (5.2\% per the NINDS-TPA trial definition), ${ }^{17}$ and the Taiwanese study (6.7\% per the SIT-MOST definition). However, since the individual studies differed in their SICH definitions as well as baseline characteristics, the observed SICH rates across the studies could not be directly compared. In a recent study of the CRCS-5 registry of Korea, the SICH rate of 1,698 patients (mean age of $67.8 \pm 13.0$ ) after intravenous TPA was $5.6 \%{ }^{20}$ Therefore, the risk of SICH does not seem to substantially and unacceptably increase in very elderly Korean patients. Furthermore, in the PS-matched cohort, the increased SICH risk did not lead to an increase in the proportion of mRS 5-6 outcome at discharge (34.3\% in the TPA group vs. $35.8 \%$ in the no-TPA group) as well as in-hospital mortality ( $8.5 \%$ in the TPA group versus $9.6 \%$ in the no-TPA group) (Figure 3 ). The pooled analysis of RCTs also showed no significant association between age and fatal intracranial hemorrhage. ${ }^{13}$ Therefore, the increased risk of SICH should not be a reason for avoiding the use of TPA in very elderly patients. However, given the 4 - to 5 -fold increased risk of SICH with TPA in elderly patients, treatment decision should be carefully made after taking into account the benefit 
and risk of the treatment. In addition, the predictors of SICH in elderly patients need to be explored in future studies.

This study is subject to several limitations. First, our findings were driven from observational registry data and thereby a risk of bias from unmeasured or residual confounders exists despite the covariates adjustment and PS matching techniques. Second, the onset-to-door time was significantly different between the TPA and non-TPA groups. Comparing outcomes among patients admitted within 4.5 hours would be more desirable. However, because of the limited number of patients admitted within 4.5 hours to ensure a sufficient sample for PS matching, more wide time window was applied to selecting control patients. Third, we used $\mathrm{mRS}$ outcome at discharge rather than at 3 months, a preferred and recommended time point for an outcome study of acute stroke therapy. However, mRS at day $7 / 10$ strongly correlated with the 90-day mRS. ${ }^{25}$ In addition, the TPA effect on the distribution of mRS scores at discharge in this study was consistent with and comparable to that on the 3-month mRS score distribution (data available for more than $80 \%$ of patients). Finally, the current results were obtained from stroke centers that were well experienced with TPA treatment and had a relatively high thrombolytic therapy rate of $13 \%$ among patients with ischemic stroke. ${ }^{20}$ Therefore, there would be a limitation in the generalizability of our findings to patients treated in less experienced centers.

\section{Conclusion}

Despite the increasing health care burden of very elderly patients, these patients have been excluded from or under-recruited in clinical trials across a wide range of conditions including stroke and other neurological diseases as well as non-neurological diseases. ${ }^{26-28}$ The lack of firm evidence from individual clinical trials has been an obstacle for an effective intervention such as TPA for acute ischemic stroke to be approved from a regulatory authority and applied to routine clinical practice in the management of very elderly patients. The current study using a well-designed prospective registry database shows that intravenous TPA within 4.5 hours improved the functional outcome despite an increased risk of SICH in very elderly Korean stroke patients. The findings, consistent with those from pooled analysis of RCTs and other observation studies, strongly support the use of TPA for the very elderly population.

\section{References}

1. Jung KH, Lee SH, Kim BJ, Yu KH, Hong KS, Lee BC, et al. Secular trends in ischemic stroke characteristics in a rapidly devel- oped country: results from the Korean Stroke Registry Study (secular trends in Korean stroke). Circ Cardiovasc Qual Outcomes 2012;5:327-334.

2. Rothwell PM, Coull AJ, Giles MF, Howard SC, Silver LE, Bull LM, et al. Change in stroke incidence, mortality, case-fatality, severity, and risk factors in Oxfordshire, UK from 1981 to 2004 (Oxford Vascular Study). Lancet 2004;363:1925-1933.

3. Marini C, Baldassarre M, Russo T, De Santis F, Sacco S, Ciancarelli I, et al. Burden of first-ever ischemic stroke in the oldest old: evidence from a population-based study. Neurology 2004; 62:77-81.

4. Di Carlo A, Baldereschi M, Gandolfo C, Candelise L, Ghetti A, Maggi $S$, et al. Stroke in an elderly population: incidence and impact on survival and daily function. The Italian Longitudinal Study on Aging. Cerebrovasc Dis 2003;16:141-150.

5. Hong KS, Bang OY, Kang DW, Yu KH, Bae HJ, Lee JS, et al. Stroke statistics in Korea: part I. Epidemiology and risk factors: a report from the Korean Stroke Society and Clinical Research Center for Stroke. J Stroke 2013;15:2-20.

6. Palm F, Urbanek C, Rose S, Buggle F, Bode B, Hennerici MG, et al. Stroke incidence and survival in Ludwigshafen am Rhein, Germany: the Ludwigshafen Stroke Study (LuSSt). Stroke 2010; 41:1865-1870.

7. Hacke W, Kaste M, Fieschi C, Toni D, Lesaffre E, von Kummer $\mathrm{R}$, et al. Intravenous thrombolysis with recombinant tissue plasminogen activator for acute hemispheric stroke. The European Cooperative Acute Stroke Study (ECASS). JAMA 1995;274: 1017-1025.

8. Hacke W, Kaste M, Fieschi C, von Kummer R, Davalos A, Meier D, et al. Randomised double-blind placebo-controlled trial of thrombolytic therapy with intravenous alteplase in acute ischaemic stroke (ECASS II). Second European-Australasian Acute Stroke Study Investigators. Lancet 1998;352:1245-1251.

9. Hacke W, Kaste M, Bluhmki E, Brozman M, Dávalos A, Guidetti D, et al. Thrombolysis with alteplase 3 to 4.5 hours after acute ischemic stroke. NEngl J Med 2008;359:1317-1329.

10. Clark WM, Wissman S, Albers GW, Jhamandas JH, Madden KP, Hamilton S. Recombinant tissue-type plasminogen activator (Alteplase) for ischemic stroke 3 to 5 hours after symptom onset. The ATLANTIS Study: a randomized controlled trial. Alteplase Thrombolysis for Acute Noninterventional Therapy in Ischemic Stroke. JAMA 1999;282:2019-2026.

11. Tissue plasminogen activator for acute ischemic stroke. The National Institute of Neurological Disorders and Stroke rt-PA Stroke Study Group. N Engl J Med 1995;333:1581-1587.

12. The IST-3 Collaborative Group. The benefits and harms of intravenous thrombolysis with recombinant tissue plasminogen activator within $6 \mathrm{~h}$ of acute ischaemic stroke (the third inter- 
national stroke trial [IST-3]): a randomised controlled trial. Lancet 2012;379:2352-2363.

13. Emberson J, Lees KR, Lyden P, Blackwell L, Albers G, Bluhmki E, et al. Effect of treatment delay, age, and stroke severity on the effects of intravenous thrombolysis with alteplase for acute ischaemic stroke: a meta-analysis of individual patient data from randomised trials. Lancet 2014;384:1929-1935.

14. Bhatnagar P, Sinha D, Parker RA, Guyler P, O’Brien A. Intravenous thrombolysis in acute ischaemic stroke: a systematic review and meta-analysis to aid decision making in patients over 80 years of age. J Neurol Neurosurg Psychiatry 2011;82:712-717.

15. Mishra NK, Ahmed N, Andersen G, Egido JA, Lindsberg PJ, Ringleb PA, et al. Thrombolysis in very elderly people: controlled comparison of SITS International Stroke Thrombolysis Registry and Virtual International Stroke Trials Archive. BMJ 2010;341:c6046.

16. Mehta RH, Cox M, Smith EE, Xian Y, Bhatt DL, Fonarow GC, et al. Race/Ethnic differences in the risk of hemorrhagic complications among patients with ischemic stroke receiving thrombolytic therapy. Stroke 2014;45:2263-2269.

17. Matsuo R, Kamouchi M, Fukuda H, Hata J, Wakisaka Y, Kuroda J, et al. Intravenous thrombolysis with recombinant tissue plasminogen activator for ischemic stroke patients over 80 years old: the Fukuoka Stroke Registry. PLoS One 2014;9:e110444.

18. Sung PS, Chen CH, Hsieh HC, Fang CW, Hsieh CY, Sun YT, et al. Outcome of acute ischemic stroke in very elderly patients: is intravenous thrombolysis beneficial? Eur Neurol 2011;66: 110-116.

19. Hong KS, Bang OY, Kim JS, Heo JH, Yu KH, Bae HJ, et al. Stroke statistics in Korea: part II stroke awareness and acute stroke care, a report from the Korean Stroke Society and Clinical Research Center for Stroke. J Stroke 2013;15:67-77.

20. Kim BJ, Park JM, Kang K, Lee SJ, Ko Y, Kim JG, et al. Case characteristics, hyperacute treatment, and outcome information from the clinical research center for stroke-fifth division registry in South Korea. J Stroke 2015;17:38-53.

21. Kim BJ, Han MK, Park TH, Park SS, Lee KB, Lee BC, et al. Current status of acute stroke management in Korea: a report on a multicenter, comprehensive acute stroke registry. Int J Stroke 2014;9:514-518.

22. Adams HP Jr, Bendixen BH, Kappelle LJ, Biller J, Love BB, Gordon DL, et al. Classification of subtype of acute ischemic stroke. Definitions for use in a multicenter clinical trial. TOAST. Trial of Org 10172 in Acute Stroke Treatment. Stroke 1993;24:35-41.

23. Samuels OB, Joseph GJ, Lynn MJ, Smith HA, Chimowitz MI. A standardized method for measuring intracranial arterial stenosis. AJNR Am J Neuroradiol 2000;21:643-646.

24. Austin PC, Mamdani MM. A comparison of propensity score methods: a case-study estimating the effectiveness of post-AMI statin use. Stat Med 2006;25:2084-2106.

25. Ovbiagele B, Saver JL. Day-90 acute ischemic stroke outcomes can be derived from early functional activity level. Cerebrovasc Dis 2010;29:50-56.

26. Sanossian N, Ovbiagele B. Prevention and management of stroke in very elderly patients. Lancet Neurol 2009;8:1031-1041.

27. Brodie MJ, Elder AT, Kwan P. Epilepsy in later life. Lancet Neurol 2009;8:1019-1030.

28. Townsley CA, Selby R, Siu LL. Systematic review of barriers to the recruitment of older patients with cancer onto clinical trials. J Clin Oncol 2005;23:3112-3124. 